\title{
Slender Delta Wing with Conical Camber
}

\author{
Rajendra K. Bora*
}

National Aeronautical Laboratory, Bangalore, India

THE slenderness assumption of Munk $^{1}$ and Jones, ${ }^{2}$ made over and above that of linearization, has often been used to get closed form analytical solutions for wings, bodies, and their combinations. In this Note, simple expressions are obtained for the aerodynamic characteristics of a slender delta wing, exhibiting an even-order polynomial twist distribution in the spanwise coordinate. In Ref. 3 is derived an integral equation which relates the twist distribution to the pressure distribution. For the slender wing it is

$$
\begin{aligned}
\Delta C_{p}=C_{p}^{-} & -C_{p}^{+} \\
& =\frac{4 C_{0}}{\tan \delta\left(1-\eta^{2}\right)^{1 / 2}}+\frac{4 \tan \delta}{\pi} \int_{0}^{1} \frac{d \omega}{d \eta_{i}} \eta_{i} \\
& \times \log \frac{\left(1-\eta^{2}\right)^{1 / 2}+\left(1-\eta_{i}{ }^{2}\right)^{1 / 2}}{\left(1-\eta^{2}\right)^{1 / 2}-\left(1-\eta_{i}{ }^{2}\right)^{1 / 2}} d \eta_{i}
\end{aligned}
$$

and

$$
C_{0}=\tan ^{2} \delta\left[-\omega(1)+\frac{2}{\pi} \int_{0}^{1} \frac{d \omega}{d \eta} \sin ^{-1} \eta d \eta\right]
$$

The twist distribution under consideration is of the type

$$
\alpha_{n}(\eta)=-\omega_{n}(\eta)=-\epsilon_{0 n}-\epsilon_{n} \eta^{2 n / 2 n}
$$

Here $C_{p}$ is the pressure coefficient, $\eta$ the conical coordinate $y / s(x), \alpha$ the twist distribution, $s(x)$ the local wing semispan, 6 the wing semiapex angle, and + and - refer to the top and bottom surfaces of the wing, respectivey. The coordinate system is shown in Fig. 1 .

Linearized theory predicts two kinds of solutions. One in which a leading edge singularity appears and the other in which the leading edge experiences zero load. Because 


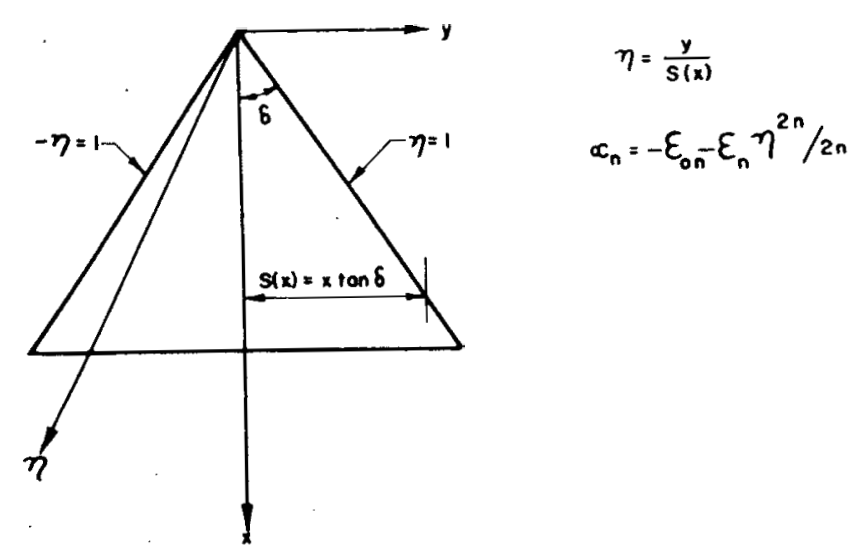

Fig. 1 Coordinate system.

the flow has a tendency to separate with the former type of flow, the latter type of solutions have been considered by several authors ${ }^{4-6}$ to design wings of low drag. We also restrict the present analysis to this case and accordingly put $C_{o}=0$. This will, therefore, 'relate the constants $\epsilon_{o n}$ and $\epsilon_{n}$.

On substituting Eq. (3) into Eq. (1) and integrating by parts, and writing down the integrals in terms of the Glauert integrals, we obtain

$$
\Delta C_{B n}=\frac{4 \tan \delta}{2 n+1} \epsilon_{n}\left(1-\eta^{2}\right)^{1 / 2} \sum_{i=0}^{n} J_{i} \eta^{2(n-i)}
$$

where

$$
\left.\begin{array}{l}
J_{0}=1 \\
\left.J_{i}=\frac{1 \cdot 3 \cdots(2 i-1)}{2 \cdot 4 \cdots(2 i)}\right\}
\end{array}\right\}
$$

and from Eq. (2)

$$
\epsilon_{0 n}=-\frac{-}{2 n n} \quad R \cdot \begin{gathered}
2 n+1 \\
2
\end{gathered}, \frac{1}{2}
$$

where $B$ is the complete Beta function and is expressible in terms of the Gamma functions.

The spanwise distribution of lift is

$$
\begin{aligned}
& \mathrm{L}_{\mathrm{n}}(\eta)=\int^{1} \Delta C_{p_{m}}\left(\eta_{i}\right) d x=\eta \int^{1} \Delta C_{D} \frac{d \eta_{i}}{n} \\
& =\frac{2 \tan \delta}{n^{\prime}(2 n+1)} \quad\left(1-\eta^{2}\right)^{3 / 2} \sum_{m=0}^{n-1}(2 m+1) J_{m} \eta^{2(n-m-1)}
\end{aligned}
$$

Notice here that unlike the plane delta which shows infinite slope for $L(\eta)$ at the wing tip, Eq. (7) shows zero slope.

The lift and drag coefficients also reduce to simple expressions. These are

$$
\begin{array}{ll} 
& C_{L_{\mathbf{n}}}=-\int_{0}^{1} A C_{b_{n}} d q \\
& =-\frac{\pi \tan \delta}{(2 n+1)} \epsilon_{n} \sum_{k=0}^{n} \frac{J_{k} J_{n-k}}{(n-k+1)} \\
\text { and } \quad & \int_{0}^{1} \Delta C_{p_{n}} \omega_{m} d \eta \\
C_{D_{n} m}= & -\epsilon_{n} \epsilon_{m} \frac{\pi \tan \delta}{(2 n+1)} \sum_{k=0}^{n} \frac{J_{k} J_{n+m-k}}{(n+m-k+1)} \\
& -\epsilon_{0 m} C_{L_{n}}
\end{array}
$$

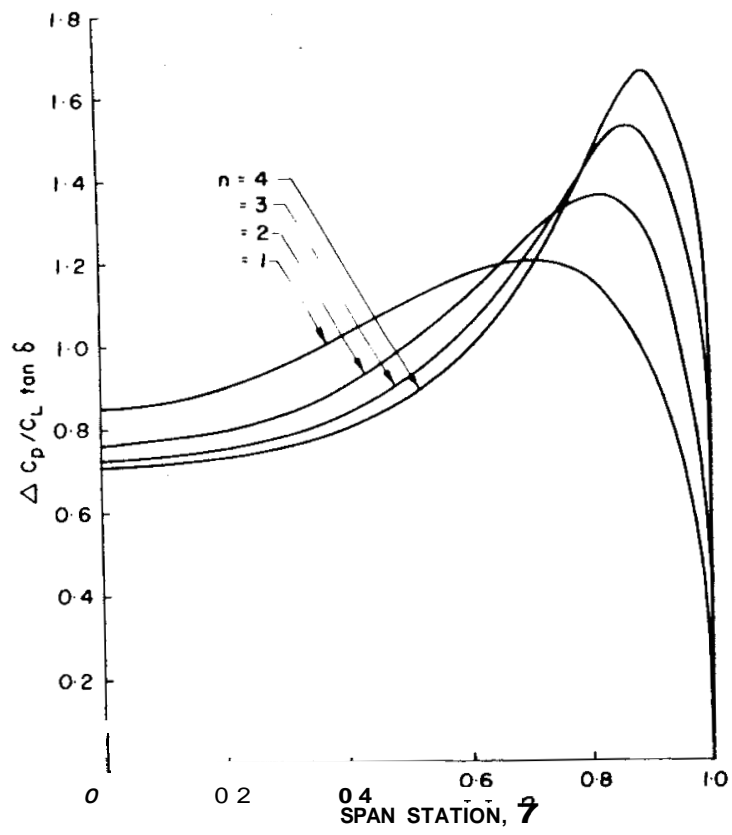

Fig. 2a Pressure distribution on slender delta with attachment line at the leading edge.

For a delta wing the chordwise distribution of lift will be linear and the centre of nressire ie firas at $4 / 3$ root chord from the wing apex. The pitching moment coefficient about the wing apex is

$$
C_{M_{n}}=-2 / 3 C_{L_{n}}
$$

The wing ordinate distribution is given by

$$
\frac{\partial z}{\partial \eta}=\bar{z}-\eta \frac{\partial \vec{z}}{\partial \eta}=\omega(\eta)
$$

where

$$
z=x \bar{z}(\eta)
$$

Integrating (11) gives

$$
\bar{z}(\eta)=K \eta-\eta \int \frac{\omega(\eta)}{\eta^{2}} d \eta
$$

where $K$ is an arbitr - cunstant anu snuws that the wing shapes are nonunique w the extent that a dihedral angle does not affect the anrodvr nis characteristics in the linearized theory. Without any loss of generality, we put $=0$, and the substitution of Eq. (3) inco $\mathrm{E \theta}$. (12) ine\# yields

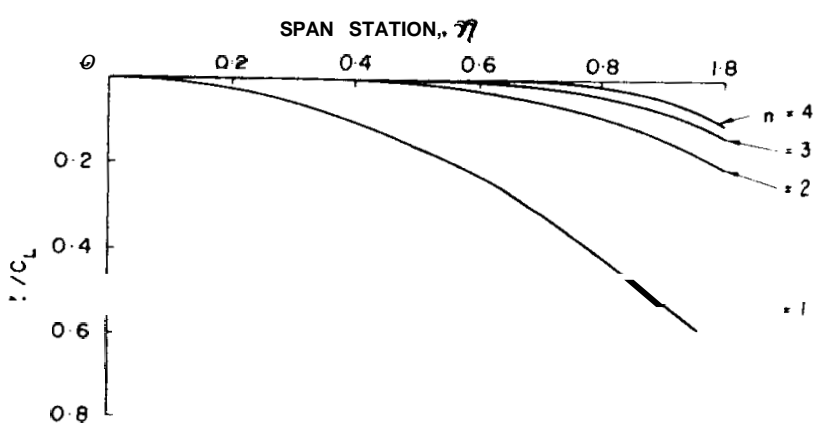

Fig. 2b Ordinate distribution of slender delta. 
These results may be extended to nondelta planforms in the same way as described by Jones. ${ }^{2}$ However, it is always useful to remember the drawbacks of any analysis when several idealizations have been made to arrive at simple solutions. Thus the slenderness assumption violates the Kutta condition in subsonic flow b y ignoring the presence of the trailing edge. As a consequence, the lift near the trailing edge is considerably overestimated. An emperical correction suggested by Laidlaw and $\mathrm{Hsu}^{7}$ when used with the slender wing results was found to be very useful and is therefore repeated here

$$
\Delta C_{p_{c}}(x, \eta)=\sqrt{1-\left(\frac{x}{C}\right)^{2}} \Delta C_{p_{i}}
$$

where $\Delta C_{p c}$ is the corrected value, $\Delta C_{p i}$ is from slender wing theory, and $c$ is the local chord. The result is valid for steady and unsteady cases and has been successfully used in flutter analysis. In supersonic flow, the presence of the trailing edge is felt only through the boundary layer and for most purposes it is sufficient to assume that pressure equalization takes place through an oblique shock. The slender wing solution is therefore valid quite close to the trailing edge. The slender theory, however, fails to predict the wave drag.

As a matter of interest, the spanwise pressure and ordinate distributions are shown in Fig. 2 for $n=1-4$. It is seen that these wings have a leading edge droop which helps the flow to turn smoothly, and hence, the predicted aerodynamic properties may be expected to be close to reality.

The analytical results of this note augment the solutions of Ref. 2 for the flat delta, and of Ref. 3 for the delta with a linear twist distribution. The simplicity of the results along with the Laidlaw-Hsu correction should make them useful where quick estimates of the pressure distribution are required.

\section{References}

1Munk, M. M., "The Aerodynamic Forces on Airship Hulls," Rept. 184,1924,NACA.

'Jones, R. T., "Properties of Low Aspect Ratio Pointed Wings at Speeds Above and Below the Speed of Sound," Rept. 835, 1946, NACA.

${ }^{3}$ Carafoli, E., High Speed Aerodynamics, Pergamon Press, New York, 1956,Chap. 9, p. 622.

'Smith, J. H. B. and Mangler, K. W., "The Use of Conical Camber to Produce Flow Attachment at the Leading Edge of a Delta Wing and to Minimize Lift Dependent Drag at Sonic and Supersonic Speeds," R and M 3289, 1957, Aeronautical Research Council, London, England.

'Tsien, S. H., "The Supersonic Conical Wing of Minimum Drag," Journal of Aircraft, Vol. 22, No. 12, Dec. 1955, pp. 805817.

"Brebner, G. G., "Some Simple Conical Camber Shapes to Produce Low Lift Dependent Drag on Slender Delta Wing," CP 428, 1957, Aeronautical Research Council, London, England.

"Laidlaw, W. R. and Hsu, P. T., "A SemiempericalMethod for Determining Delta Wing Pressure Distributions in an Incompressible Flow," Journal of the Aeronautical Sciences, Vol. 21, No. 12, Dec. 1954 , pp. $854-856$. 